\title{
Cytoreductive surgery and hyperthermic intraperitoneal chemotherapy in gastrointestinal cancers: fad or standard of care?
}

Melissa Ching Ching $\underline{T e O}^{1}$, MBBS, FRCS, Grace Hwei Ching $\underline{\operatorname{Tan}}^{1}$, MBBS, FRCS

\begin{abstract}
Peritoneal metastases (PM) are the common endpoint for patients with advanced gastrointestinal cancers. PM from these cancers are often managed in a similar fashion to other sites of systemic metastases, but the following must be taken into consideration. (a) PM do not respond to systemic chemotherapy in the same fashion as liver and lung metastases. (b) PM cause local problems, resulting in disruption of chemotherapy. (c) Cytoreductive surgery and hyperthermic intraperitoneal chemotherapy (CRS-HIPEC) actually work for PM. (d) PM are not easily detected on imaging modalities. There has been mounting evidence of the effectiveness of CRS-HIPEC at prolonging survival in selected patients with colorectal and gastric PM, but there remains a reluctance to explore this treatment modality. This is likely because of the perceived morbidity and mortality. An effective management strategy employing CRS-HIPEC for selected patients with gastrointestinal PM can only be achieved if a concerted effort is made to understand this disease and address the concerns regarding this treatment.
\end{abstract}

Keywords: CRS, HIPEC, peritoneal metastases

\section{INTRODUCTION}

Cytoreductive surgery (CRS) and hyperthermic intraperitoneal chemotherapy (HIPEC) have been utilised with significant success since 1994 for the management of pseudomyxoma peritonei, with its widespread peritoneal involvement. ${ }^{(1)}$ Since then, researchers in various institutions have explored the potential benefit that this combined treatment may offer for other diseases involving the peritoneal surfaces. In specialised centres that have overcome the learning curve, CRS and HIPEC are routinely performed for suitable patients who have peritoneal metastases (PM) with gastrointestinal and gynaecological origins. However, a large majority of physicians and surgeons remain sceptical and continue to manage patients with palliative surgical procedures and/or palliative chemotherapy and, in some situations, the best supportive care. This discrepancy between approaches often leads to confusion for patients who seek multiple opinions in their search for the best treatment option for their condition. It is important to examine the evidence and separate the facts from myths and hearsay, to determine if CRS and HIPEC for the management of PM from gastrointestinal cancers is a fad or should be the standard of care.

\section{WHAT ARE CRS AND HIPEC?}

CRS and HIPEC comprise two parts of a single treatment modality. CRS was first described by Spratt et al in $1980^{(2)}$ and popularised by Sugarbaker in 1995.(3) It aims to remove all macroscopic peritoneal disease; resection of involved visceral organs is typically performed first, followed by the removal of sections of involved peritoneum. HIPEC targets the microscopic diseases, working on lesions that are smaller than $3 \mathrm{~mm}$. Owing to the peritoneal-plasma barrier, a higher dose of chemotherapy can be delivered with less systemic toxicity. The high temperature increases drug penetration and provides a synergistic effect with the intraperitoneal chemotherapy. HIPEC is administered for 60 minutes. A dedicated anaesthetist monitors the patient's parameters, including core temperature, via an oesophageal temperature probe and keeps the patient adequately volume-filled. We herein examine the evidence in favour of the use of CRS and HIPEC for the common gastrointestinal cancers.

\section{Colorectal cancer}

Colorectal cancer is one of the commonest cancers in most developed countries, has a worldwide incidence of 43.7 per 100,000 men and women per year ${ }^{(4)}$ and afflicts one in 20 in the United States alone. ${ }^{(5)}$ Despite efforts to conduct screening, $60 \%$ of patients are diagnosed at an advanced stage with a significantly poorer prognosis compared to early-stage disease, resulting in close to 50,000 cancer deaths per year. ${ }^{(5)}$

Cancer deaths in Stage 4 colorectal cancer often result from liver, lung or peritoneal metastases, or a combination of disease at these sites. Traditionally, the prognosis is dismal for most Stage 4 cancers. However, complete cytoreduction along with adjuvant chemotherapy has provided long-term survival of up to $40 \%$ and $50 \%$ for patients with lung and liver metastases, respectively. ${ }^{(6-9)}$ As such, consideration for curative therapy is now accepted as the standard of care for patients with metastases at such sites, and it is routine for these patients to be discussed at multidisciplinary tumour boards. . $^{(8,9}$

PM are diagnosed synchronously in $10 \%-15 \%$ of all newly diagnosed colorectal cancer cases and in $40 \%-70 \%$ of patients who suffer recurrences. They are the sole sites of metastases in $10 \%-30 \%$ of these cases, but the approach to the management of colorectal cancer PM remains a point of debate. The Fourth 
International Workshop on Peritoneal Surface Malignancies in Spain came to a consensus in 2004 that "cytoreductive surgery with perioperative intraperitoneal chemotherapy was considered standard of care for all cases of mucinous appendiceal neoplasms with peritoneal dissemination, in an otherwise fit patient in the absence of distant metastases" ${ }^{\prime 10)}$ Another consensus statement was issued at the 59th Annual Cancer Symposium of the Society of Surgical Oncology held in San Diego in 2006, stating that "Better surgical techniques that include peritonectomy procedures, standardised methods to deliver intraoperative hyperthermic intraperitoneal chemotherapy and better patient selection criteria have resulted in a significant improvement in survival and in morbidity and mortality of the surgical management of this particular group of Stage IV colon cancer patients" ${ }^{(11)}$ Despite the lapse of ten years since these statements have circulated, and in spite of the large volume of publications and mounting evidence that CRS and HIPEC afford long-term survival in selected patients with colorectal PM, this treatment modality is still not universally considered the standard of care and many patients are routed to a palliative course, usually with chemotherapy.

The role of CRS and HIPEC for colorectal PM was established in the first randomised prospective trial in 2003. ${ }^{(12)}$ In the trial, 105 patients were assigned to either systemic chemotherapy (5-fluorouracil/leucovorin) with or without palliative surgery, or CRS and HIPEC with mitomycin C, followed by systemic chemotherapy. Median survival times of 12.6 months and 22.3 months in the standard treatment arm and the CRS and HIPEC arm, respectively $(p=0.032)$, were reported in the preliminary study. The CRS and HIPEC arm also reported an associated mortality rate of $8 \%$. The 2008 update of the study reported disease-specific survival times of 12.6 months and 22.2 months in the control and the CRS and HIPEC arms, ${ }^{(13)}$ respectively. The trial has received much criticism, most notably concerning the high mortality rate experienced by those in the CRS and HIPEC arm, and also the somewhat outdated chemotherapy regime used in the standard arm. A much larger and more recent study on the outcomes of CRS and HIPEC for colorectal PM by Glehen et $\mathrm{al}^{(14)}$ involved 506 patients treated at 28 institutions. Morbidity and mortality rates of $22.9 \%$ and $4 \%$, respectively, and overall survival (OS) time of 19.2 months were attained.

\section{Gastric cancer}

PM occur in up to $70 \%$ of gastric cancer patients. They are found intraoperatively during planned surgical resection in $10 \%-20 \%$ of patients, will develop in $20 \%-50 \%$ of patients after curative surgery $^{(15)}$ and are the cause of death in $60 \%$ of gastric cancer patients. ${ }^{(16)}$ Traditionally, PM with a gastric origin are considered distant metastases. Often, these patients are treated with a palliative intent and have a median survival time of 3-6 months. ${ }^{(17)}$ Palliative treatment often includes systemic chemotherapy or best supportive care, and studies have shown a survival benefit from systemic chemotherapy for patients with advanced gastric cancer. ${ }^{(18)}$ However, these studies notably included all metastatic gastric cancer patients and did not reflect the minimal effect of systemic chemotherapy on the peritoneal disease. ${ }^{(19,20)}$
In recent years, CRS and intraperitoneal chemotherapy have been proposed to treat selected patients with gastric peritoneal metastases (GPM), with variable results. ${ }^{(21)}$ Currently, intraperitoneal chemotherapy can be given in three ways: (a) HIPEC; (b) neoadjuvant intraperitoneal/systemic (bidirectional) chemotherapy (NIPS); and (c) early postoperative intraperitoneal chemotherapy (EPIC).

\section{CRS and HIPEC}

CRS alone has shown poor results when it was attempted for GPM, (21) but has had promising outcomes when combined with HIPEC. One caveat for CRS is that it is more effective for patients with a low disease burden, with patients who had a peritoneal cancer index score of $<7$ benefitting from the procedure. ${ }^{(22)}$

CRS and HIPEC have been employed in two different settings. In the first, patients with early gastric cancers received the treatment as an adjuvant and a prophylactic. In the second, advanced gastric cancer patients underwent the procedure as a cure. Yonemura et $\mathrm{al}^{(23)}$ randomised 139 patients with Stage 2-4 gastric cancer into three groups: HIPEC and surgery, intraperitoneal normothermic chemotherapy plus surgery, and surgery alone. The results indicated that the group that received HIPEC had an improved survival rate of $61 \%$, while the intraperitoneal normothermic chemotherapy plus surgery and the surgery alone groups had survival rates of $43 \%$ and $42 \%$, respectively. Similar results have been shown by other Japanese groups, with five-year OS rates of up to $30 \%{ }^{.24)}$ In a French study, a five-year survival rate of $23 \%$ was reported in patients with Stage 3-4 gastric cancer, and only when complete cytoreduction was achieved. ${ }^{(25)}$

\section{Neoadjuvant intraperitoneal/systemic chemotherapy}

NIPS, the latest development in intraperitoneal chemotherapy, is a bidirectional chemotherapy that targets PM from both sides of the peritoneum (the peritoneal cavity and subperitoneal blood vessels). The rationale is to downstage the disease, improve the likelihood of complete cytoreduction and, together with CRS and HIPEC, achieve improved outcomes. Yonemura et al have been the pioneer in this area, showing remarkable response rates of up to $69 \%$. ${ }^{(22)}$ There are, however, some downsides to this therapy, as the literature shows the risks of high toxicity and the heterogeneity of systemic and intraperitoneal chemotherapy regimes.

\section{Early postoperative intraperitoneal chemotherapy}

EPIC is administered in the first few days after CRS with the aim of eradicating residual intraperitoneal cancer cells in the postoperative period, before the development of adhesions. In an early study by Jeung et al, the administration of EPIC resulted in an OS time of 12 months for patients with intra-abdominal gross residual lesions after palliative gastrectomy. ${ }^{(26)}$ The Korean group showed that patients who received EPIC after gastric resection had improved OS compared to patients who had surgery only. The results were particularly striking for patients with Stage III and IV gastric cancer. ${ }^{(27)}$ 


\section{Small bowel cancer}

Small bowel cancer is much more uncommon compared to colorectal and gastric cancers, and constitutes only $0.6 \%$ of all newly diagnosed cancers in the United States, afflicting less than 10,000 people annually based on 2014 data. ${ }^{(28)}$ As with the other gastrointestinal cancers, the peritoneum is not spared from metastases in small bowel cancer and the prognosis is uniformly poor once such a diagnosis is reached.

A few publications have described the use of CRS and HIPEC for PM from small bowel cancer and provided a similar underlying principle and rationale for the choice of this combined treatment modality. ${ }^{(29,30)}$ Van Oudheusden et al reported that CRS and HIPEC were successfully performed in $84 \%$ of patients who underwent explorative laparotomy, and that morbidity and mortality rates were $25 \%$ and $0 \%$, respectively. ${ }^{(29)}$ Disease recurrence was $50 \%$ in his series of 19 patients and median survival time was 31 months. ${ }^{(29)}$ In another paper on CRS and HIPEC for gastrointestinal cancers, where two out of 22 patients were being treated for PM from small bowel cancer, the two-year survival rate was reportedly $46 \%$ and significant prognostic factors included completeness of resection and peritoneal carcinomatosis index (PCI) scores. ${ }^{(30)}$ However, the use of CRS and HIPEC for small bowel cancer is still considered experimental, and the tumour biology is likely to be different from that of colorectal and gastric cancers, which will be a limiting factor. The role of CRS and HIPEC in the management of small bowel peritoneal disease can perhaps be considered in the context of a clinical trial.

\section{AS FIRST CONSIDERATION FOR ISOLATED PERITONEAL METASTASES} Poor response to systemic chemotherapy

Patients with colorectal metastases are usually administered chemotherapy in a bid to control the systemic disease. Upfront chemotherapy or chemotherapy after the resection of the primary tumour but before the metastatectomy (pseudo-neoadjuvant chemotherapy) in those who present with synchronous metastases is often employed for a twofold purpose: (a) to allow the determination of the specific biology of that particular tumour and (b) for an in vivo test of the efficacy of the chemotherapeutic agent for that tumour. Patients whose tumours respond to the prescribed chemotherapy are often considered for resection of the metastases, especially if these are situated in the liver or lung, and complete resection with clear margins is deemed possible. In patients who present with metachronous lesions, upfront resection is often considered for liver and lung metastases, especially if there has been a reasonable disease-free interval and the patient has not progressed on adjuvant chemotherapy. ${ }^{(7,9)}$ This approach has allowed improved prognoses among patients with resectable liver and lung metastases.

However, the situation is quite different for patients with PM, whose disease often does not respond to systemic chemotherapy. In many publications about patients on palliative chemotherapy for colorectal metastases, survival data is based on treatment of metastases at all sites. ${ }^{(31)}$ There remain few reports of similar evidence in patients with PM being treated with chemotherapy.
In Jayne et al's study looking at this issue, it was found that a diagnosis of PM immediately confers a poorer prognosis by threefold, and that the median OS is seven months. ${ }^{(32)}$ Hence, the often-quoted prolonged survival with state-of-the-art chemotherapy for colorectal metastases cannot be extrapolated to those with peritoneal disease.

It is not clear why PM respond differently to systemic chemotherapy compared to liver and lung metastases; however, chemotherapy-induced toxicity causing early discontinuation, the poorer vasculature of the peritoneum, and an unfavourable biological profile of these tumours have been postulated as being reasons for the difference. ${ }^{(33-35)}$ Further molecular studies on response to chemotherapy at different metastatic sites would shed more light on the matter, but investigations are still ongoing. Currently, targeted agents are increasingly being utilised in the treatment of Stage 4 gastrointestinal cancers, but in conjunction with standard chemotherapy, and hence result in similar issues of systemic toxicity.

\section{Local problems disrupting planned chemotherapy}

It is often not difficult to spot and diagnose a patient with peritoneal disease, as these patients are often symptomatic, with symptoms of abdominal distension and bloatedness, shortness of breath and poor oral intake. Some present with respiratory difficulties secondary to splinting of the diaphragm from massive ascites, requiring insertion of cope loop catheters for drainage of the ascites and temporary relief. These patients are frequently referred to general surgeons by medical oncologists when they experience symptoms of intestinal obstruction, either in the midst of chemotherapy or while they are being managed conservatively. Patients with intestinal obstruction often require admission for bowel rest, intravenous hydration and, in some instances, a palliative surgical procedure of resection or bypass of the offending obstructive lesion. In comparison, most patients with liver or lung metastases remain asymptomatic and are able to complete their planned chemotherapy. On evaluation of our institution's data (to be published), it was found that only $29 \%$ of patients with PM from colorectal cancer were able to complete their planned courses of palliative chemotherapy. ${ }^{(36)}$

\section{Effectiveness for peritoneal disease}

Multiple articles have been published since Verwaal et al's randomised trial on CRS and HIPEC versus intravenous chemotherapy for PM from colorectal cancer. ${ }^{(12)}$ Prolonged survival in the arm treated with CRS and HIPEC has been similarly reported in Glehen's review ${ }^{(14)}$ following the earlier publications. To date, state-of-the-art chemotherapy has not been able to attain such long-term survival results.

Several recent cohort studies have continued to show a survival benefit for patients with PM for colorectal cancer. These include an Italian group's ten-year experience, which reported an OS of 24.6 months, with $\mathrm{PCl}$ and completeness of cytoreduction as important prognostic factors affecting survival. ${ }^{(37)}$ The Peritoneal Malignancy Unit in Basingstoke, United Kingdom, also recently performed a systemic review on the same topic and concluded that enhanced survival can be achieved with CRS and HIPEC. ${ }^{(38)}$ 


\section{TREATING AN UNDETECTABLE DISEASE: A PROACTIVE APPROACH?}

Patients with gastrointestinal cancers are often kept on surveillance, with regular visits to physicians' clinics where a thorough history and physical examination are performed, along with a sampling of their serum carcinoembryonic antigen level, surveillance endoscopy and imaging. In patients who develop recurrences, the hope is for early enough detection that would enable a potentially curative approach to the management of the recurrence. This often entails a multidisciplinary approach of combination therapy using surgery and chemotherapy, which is usually a good strategy for the management of liver and lung metastases in colorectal cancers. When these sites of metastases are detected in gastric and small bowel cancers, the prognoses are uniformly poor and a palliative approach is usually taken.

The problem with peritoneal disease is that the current surveillance modalities are unable to detect it in the early stages. In fact, a significant proportion of patients who are found to have peritoneal disease on surveillance imaging are found to have significantly more disease on laparotomy. The volume of disease may determine the prognosis, as it has been found that in patients undergoing CRS and HIPEC, the PCI that describes the extent of peritoneal disease is a significant prognostic factor. The volume of disease may even preclude a potentially curative resection, especially if it is found to involve much of the small bowel serosa. The threshold for abandoning a planned CRS and HIPEC is even lower for high PCI scores in gastric and duodenal cancers, diseases with tumour biologies that are distinct from that of colorectal cancers.

In Elias et al's systematic second-look surgery in patients at high risk of developing colorectal PM, more than 50\% of these patients were found to have peritoneal disease that was not detected by clinical and imaging modalities. ${ }^{(39)}$ The high-risk features included peritoneal nodules detected at the time of the primary cancer resection, presence of ovarian metastases at primary surgery, primary cancer perforation or obstruction, adjacent organ or structure invasion, and fistula formation. In addition, histopathology played a significant role; patients with positive margins, positive peritoneal fluid cytology, T3 or T4 mucinous cancer, signet ring morphology and positive nodal status had an increased risk of PM as well. This finding has led to the advent of proactive management for high-risk colorectal cancers, and reduced rates of PM and local recurrence of $4 \%$ (vs. $22 \%$ ) in patients managed with prophylactic CRS and HIPEC compared to those who underwent complete surgical resection only. ${ }^{(40)}$ Significant differences were seen in the median overall and disease-free survival rates as well. The current practice for patients with high-risk colorectal cancers is adjuvant systemic chemotherapy after primary cancer resection. However, with the knowledge that systemic chemotherapy does not work well for peritoneal disease and that up to $50 \%$ of cases may remain undetected even in the face of a recurrence in the peritoneum, it would seem prudent that the appropriate 'adjuvant' treatment is administered in each situation and that patients who stand a high risk of developing peritoneal disease should be treated with adjuvant HIPEC instead. This rationale may be even more important for those with gastric and duodenal cancers, who often suffer a local recurrence and whose disease course often runs a more aggressive route, followed soon after by systemic metastases. The benefit of intervening early, even before the development of PM in these cancers, would be the major factor in the consideration of adjuvant HIPEC after a complete surgical resection of the primary tumour.

\section{OTHER CONSIDERATIONS}

As with every complex procedure, CRS and HIPEC have widely reported morbidity and mortality rates. These ranged from $40 \%$ to $80 \%$ and $3 \%$ to $20 \%$, respectively, in the earlier years but have since improved to $20 \%-40 \%$ and $3 \%$, respectively. ${ }^{(41)}$ Despite the improvement, many surgeons and oncologists who are unfamiliar with this treatment modality continue to quote the earlier figures, often in a bid to dissuade patients who have read about CRS and HIPEC as a potentially viable treatment option for their disease.

It has to be acknowledged that the selection process is critical and necessitates that patients have disease that is confined to the peritoneal cavity and are of fit enough status to undergo a major surgical procedure. In addition, the physician should present and discuss each case at a multidisciplinary tumour board, in order to exclude patients who would not benefit from CRS and HIPEC and to appropriately advise those who may benefit from neoadjuvant or adjuvant treatment prior to or after CRS and HIPEC, respectively. It is also important to note that completeness of resection remains the most important factor in prognosis. ${ }^{(42-44)}$

Institutions should, ideally, welcome discussion of such complex cases and decide if there is enough supporting infrastructure, fuelled by committed multidisciplinary parties, to embark on this but, as with every complex procedure, this involves a learning curve. It is possible to eventually reduce morbidity and mortality rates in experienced centres to those similar to a Whipple's resection, ${ }^{(45)}$ leading to improved survival outcomes. ${ }^{(46,47)}$ Kusamura et al's paper describes a surgical team or institution as having proficiency at CRS and HIPEC after they have completed 140 cases. ${ }^{(18,46)}$

\section{CONCLUSION}

PM are a common endpoint for many gastrointestinal cancers and often occur together with debilitating symptoms. CRS and HIPEC have been proven to improve survival outcomes with acceptable morbidity and mortality. Stakeholders, including patients and those in the oncologic community, need to understand the rationale of the procedure including the selection criteria, address the concerns and tolerate the learning curve, before CRS and HIPEC can become the standard of care for selected patients with gastrointestinal PM.

\section{REFERENCES}

1. Ansari N, Chandrakumaran K, Dayal S, et al. Cytoreductive surgery and hyperthermic intraperitoneal chemotherapy in 1000 patients with perforated appendiceal epithelial tumours. Eur J Surg Oncol 2016; 42:1035-41.

2. Spratt JS, Adcock RA, Muskovin M, Sherrill W, McKeown J. Clinical delivery 
system for intraperitoneal hyperthermic chemotherapy. Cancer Res 1980; 40:256-60.

3. Sugarbaker PH. Peritonectomy procedures. Ann Surg 1995; 221:29-42.

4. US Cancer Statistics Working Group. United States Cancer Statistics: 1999-2011 Incidence and Mortality Web-based Report. Department of Health and Human Services, Centers for Disease Control and Prevention and National Cancer Institute; 2014. Available at: http://www.cdc.gov/uscs. Accessed April 24, 2016

5. American Cancer Society. Colorectal Cancer Facts \& Figures 2014-2016. Available at: http://www.cancer.org/acs/groups/content/documents/document/ acspc-042280.pdf. Accessed April 24, 2016.

6. Rees M, Tekkis PP, Welsh FK, O'Rourke T, John TG. Evaluation of long-term survival after hepatic resection for metastatic colorectal cancer: a multifactorial model of 929 patients. Ann Surg 2008; 247:125-35.

7. Morris EJ, Forman D, Thomas JD, et al. Surgical management and outcomes of colorectal cancer liver metastases. Br J Surg 2010; 97:1110-8.

8. Saito Y, Omiya H, Kohno K, et al. Pulmonary metastasectomy for 165 patients with colorectal carcinoma: a prognostic assessment. J Thorac Cardiovasc Surg 2002; 124:1007-13.

9. Tampellini M, Ottone A, Bellini E, et al. The role of lung metastasis resection in improving outcome of colorectal cancer patients: results from a large retrospective study. Oncologist 2012; 17:1430-8.

10. González-Moreno S. Peritoneal surface oncology: a progress report. Eur J Surg Oncol 2006; 32:593-6.

11. Esquivel J, Sticca R, Sugarbaker P, et al; Society of Surgical Oncology Annual Meeting. Cytoreductive surgery and hyperthermic intraperitoneal chemotherapy in the management of peritoneal surface malignancies of colonic origin: a consensus statement. Society of Surgical Oncology. Ann Surg Oncol 2007; 14:128-33.

12. Verwaal VJ, van Ruth S, de Bree E, et al. Randomized trial of cytoreduction and hyperthermic intraperitoneal chemotherapy versus systemic chemotherapy and palliative surgery in patients with peritoneal carcinomatosis of colorectal cancer. J Clin Oncol 2003; 21:3737-43.

13. Verwaal VJ, Bruin S, Boot H, van Slooten G, van Tinteren H. 8-year followup of randomized trials: cytoreduction and hyperthermic intraperitoneal chemotherapy versus systemic chemotherapy in patients with peritoneal carcinomatosis of colorectal cancer. Ann Surg Oncol 2008; 15:2426-32.

14. Glehen O, Kwiatkowski F, Sugarbaker PH, et al. Cytoreductive surgery combined with perioperative intraperitoneal chemotherapy for the management of peritoneal carcinomatosis from colorectal cancer: a multi-institutional study. J Clin Oncol 2004; 22:3284-92.

15. Montori G, Coccolini F, Ceresoli M, et al. The treatment of peritonea carcinomatosis in advanced gastric cancer: state of the Art. Int J Surg Oncol 2014; 2014:912418.

16. Wagner AD, Grothe W, Haerting J, et al. Chemotherapy in advanced gastric cancer: a systemic review and meta-analysis based on aggregate data. J Clin Oncol 2006; 24:2903-9.

17. Bertuccio P, Chatenoud L, Levi F, et al. Recent patterns in gastric cancer: a global overview. Int J Cancer 2009; 125:666-73.

18. Roth AD, Fazio N, Stupp R, et al; Swiss Group for Clinical Cancer Research. Docetaxel, cisplatin, and fluorouracil; docetaxel and cisplatin; and epirubicin, cisplatin, and fluorouracil as systemic treatment for advanced gastric carcinoma: a randomized phase II trial of the Swiss Group for Clinical Cancer Research. J Clin Oncol 2007; 25:3217-23.

19. Ross $P$, Nicolson $M$, Cunningham D, et al. Prospective randomized trial comparing mitomycin, cisplatin, and protracted venous-infusion fluorouracil (PVI 5-FU) with epirubicin, cisplatin, and PVI 5-FU in advanced esophagogastric cancer. J Clin Oncol 2002; 20:1996-2004.

20. Yabusaki Y, Nashimoto A, Tanaka O. [Evaluation of TS-1 combined with cisplatin for neoadjuvant chemotherapy in patients with advanced gastric cancer]. Gan To Kagaku Ryoho 2003; 30:1933-40. Japanese.

21. Yonemura $Y$, Endou $Y$, Sasaki $T$, et al. Surgical treatment for peritoneal carcinomatosis from gastric cancer. Eur J Surg Oncol 2010; 36:1131-8.

22. Yonemura Y, Elnemr A, Endou Y, et al. Multidisciplinary therapy for treatment of patients with peritoneal carcinomatosis from gastric cancer. World J Gastrointest Oncol 2010; 2:85-97.

23. Yonemura Y, de Aretxabala X, Fujimura T, et al. Intraoperative chemohyperthermic peritoneal perfusion as an adjuvant to gastric cancer: final results of a randomized controlled study. Hepatogastroenterology 2001; 48:1776-82.

24. Kim JY, Bae HS. A controlled clinical study of serosa-invasive gastric carcinoma patients who underwent surgery plus intraperitoneal hyperthermo-chemo-perfusion (IHCP). Gastric Cancer 2001; 4:27-33.

25. Glehen O, Gilly FN, Arvieux C, et al. Peritoneal carcinomatosis from gastric cancer: a multi-institutional study of 159 patients treated by cytoreductive surgery combined with perioperative intraperitoneal chemotherapy. Ann Surg Oncol 2010; 17:2370-7.

26. Jeung HC, Rha SY, Jang WI, Noh SH, Chung HC. Treatment of advanced gastric cancer by palliative gastrectomy, cytoreductive therapy and postoperative intraperitoneal chemotherapy. Br J Surg 2002; 89:460-6.

27. Yu W, Whang I, Chung HY, Averbach A, Sugarbaker PH. Indication for early postoperative intraperitoneal chemotherapy for advanced gastric cancer: results of a prospective randomized trial. World J Surg 2001; 25:985-90.

28. Howlader N, Noone AM, Krapcho M, et al. SEER Cancer Statistics Review, 1975-2012. In: National Cancer Institute, Bethesda, MD. Available at: http:// seer.cancer.gov/csr/1975 2012/. Accessed April 24, 2016.

29. van Oudheusden TR, Lemmens VE, Braam HJ, et al. Peritoneal metastases from small bowel cancer: results of cytoreductive surgery and hyperthermic intraperitoneal chemotherapy in the Netherlands. Surgery 2015; 157:1023-7.

30. Shehata M, Chu F, Saunders V, et al. Peritoneal carcinomatosis from colorectal cancer and small bowel cancer treated with peritonectomy. ANZ J Surg 2006; 76:467-71

31. Vallicelli C, Cavaliere D, Catena F, et al. Management of peritoneal carcinomatosis from colorectal cancer: review of the literature. Int J Colorectal Dis 2014; 29:895-8.

32. Jayne DG, Fook S, Loi C, Seow-Choen F. Peritoneal carcinomatosis from colorectal cancer. Br J Surg 2002; 89:1545-50.

33. Franko J, Shi Q, Goldman CD, et al. Treatment of colorectal peritoneal carcinomatosis with systemic chemotherapy: a pooled analysis of north central cancer treatment group phase III trials N9741 and N9841. J Clin Oncol 2012; 30:263-7.

34. Klaver YL, Simkens LH, Lemmens VE, et al. Outcomes of colorectal cancer patients with peritoneal carcinomatosis treated with chemotherapy with and without targeted therapy. Eur J Surg Oncol 2012; 38:617-23.

35. Folprecht G, Köhne CH, Lutz MP. Systemic chemotherapy in patients with peritoneal carcinomatosis from colorectal cancer. Cancer Treat Res 2007; 134:425-40.

36. Thiruchelvam N, Chia SLC, Tan G, Teo M. Palliative Chemotherapy is a dismal option compared to CRS and HIPEC for patients with colorectal peritoneal carcinomatosis: Outcomes in a tertiary Asian institution. In press.

37. Vaira M, Cioppa T, $D^{\prime}$ amico $S$, et al. Treatment of peritoneal carcinomatosis from colonic cancer by cytoreduction, peritonectomy and hyperthermic intraperitoneal chemotherapy (HIPEC). Experience of ten years. In Vivo 2010; 24:79-84.

38. Mirnezami R, Moran BJ, Harvey K, et al. Cytoreductive surgery and intraperitoneal chemotherapy for colorectal peritoneal metastases. World J Gastroenterol 2014; 10:14018-32.

39. Elias D, Goéré D, Di Pietrantonio D, et al. Results of systematic secondlook surgery in patients at high risk of developing colorectal peritoneal carcinomatosis. Ann Surg 2008; 247:445-50.

40. Sammartino P, Sibio S, Biacchi D, et al. Long-term results after proactive management for locoregional control in patients with colonic cancer at high risk of peritoneal metastases. Int J Colorectal Dis 2014; 29:1081-9.

41. Chua TC, Yan TD, Saxena A, Morris DL. Should the treatment of peritoneal carcinomatosis by cytoreductive surgery and hyperthermic intraperitoneal chemotherapy still be regarded as a highly morbid procedure?: a systematic review of morbidity and mortality. Ann Surg 2009; 249:900-7.

42. Sugarbaker PH. Successful management of microscopic residual disease in large bowel cancer. Cancer Chemother Pharmacol 1999; 43 Suppl: S15-25.

43. Sugarbaker PH, Jablonski KA. Prognostic features of 51 colorectal and 130 appendiceal cancer patients with peritoneal carcinomatosis treated by cytoreductive surgery and intraperitoneal chemotherapy. Ann Surg 1995; 221:124-32.

44. Sugarbaker $\mathrm{PH}$. Cytoreductive surgery plus hyperthermic perioperative chemotherapy for selected patients with peritoneal metastases from colorectal cancer: a new standard of care or an experimental approach? Gastroenterol Res Pract 2012; 2012:309417.

45. Balcom JH 4th, Rattner DW, Warshaw AL, Chang Y, Fernandez-del Castillo C. Ten-year experience with 733 pancreatic resections: changing indications, older patients, and decreasing length of hospitalization. Arch Surg 2001; 136:391-8.

46. Kusamura S, Baratti D, Hutanu I, Rossi P, Deraco M. The importance of the learning curve and surveillance of surgical performance in peritoneal surface malignancy programs. Surg Oncol Clin N Am 2012; 21:559-76.

47. Polanco PM, Ding Y, Knox JM, et al. Institutional learning curve of cytoreductive surgery and hyperthermic intraperitoneal chemoperfusion for peritoneal malignancies. Ann Surg Oncol 2015; 22:1673-9. 